\title{
TOLERÂNCIA DE PLÂNTULAS DE Cedrela fissilis VELL. A DIFERENTES AMPLITUDES E INTENSIDADES DE INUNDAÇÃO
}

\author{
TOLERANCE OF Cedrela fissilis VELL. SEEDLINGS TO DIFFERENT LENGHTS AND \\ INTENSITIES OF FLOODING
}

\author{
Beatris Binotto $^{1}$ Ana Paula Antoniazzi ${ }^{2}$ Graciele Marta Neumann ${ }^{3}$ Tanise Luisa Sausen ${ }^{4}$ \\ Jean Carlos Budke ${ }^{5}$
}

\begin{abstract}
RESUMO
A presença de água no solo, tanto em regime temporário quanto permanente, exige diferentes estratégias adaptativas das espécies vegetais que ocupam estas áreas. Neste sentido, investigamos as respostas morfológicas e de alocação de biomassa em plântulas de Cedrela fissilis Vell. (Meliaceae) submetidas a diferentes amplitudes e intensidades de inundação. Plântulas de Cedrela fissilis foram submetidas a duas intensidades de inundação (submersão ao nível de coleto e total) em três diferentes amplitudes (cinco, 15 e 20 dias) e após 30, 60 e 90 dias de recuperação da condição de inundação foram realizadas avaliações da altura da parte aérea, número de folhas, diâmetro do colo e índices de clorofila $a, b$ e, total. Após 90 dias de recuperação, a altura total, diâmetro da raiz, comprimento da raiz, biomassa total, área foliar e o crescimento das raízes foram avaliados. Plântulas não inundadas constituíram um grupo controle. As plântulas submetidas à inundação total não sobreviveram. Por outro lado, as plântulas sob inundação parcial recuperaram a taxa de crescimento ao longo dos 90 dias de recuperação da inundação. Não foram observadas diferenças nas variáveis de crescimento e alocação de biomassa em relação às plântulas-controle. Todavia, observou-se que durante o período de recuperação, as plântulas submetidas a 20 dias de inundação parcial apresentaram maior taxa de abscisão foliar acompanhado por um aumento nos índices de clorofila $a$ e $b$. Os resultados indicaram que esta espécie apresenta tolerância à intensidade de inundação parcial, devendo ser cuidadosamente selecionada quanto às áreas de plantio.
\end{abstract}

Palavras-chave: alocação de biomassa; floresta ribeirinha; mudas florestais; regime hidrológico.

\begin{abstract}
The presence of water in the soil, in both temporary and permanent regimes, requires different adaptive strategies of plant species that occupy these areas. On this way, we investigated morphological responses of Cedrela fissilis Vell. (Meliaceae) seedlings subjected to different lengths and intensities of flooding. Seedlings of Cedrela fissilis were subjected to two flooding intensity treatments (up to the neck level and total submersion) in three different lengths (five, 15 and 20 days) and we assessed shoot height, numbers of leaf and leaflet, stem diameter and chlorophyll indices $a, b$ and total after 30,60 and 90 days of recovery from the flood condition. After 90 days of recovery, total height, root diameter, root size, total biomass, leaf area and root growth were evaluated. Non- flooded seedlings constituted a control group. The seedlings subjected to total flooding did not survive. On the other hand, those seedlings under partial flooding restarted

1 Bióloga, Lago Azul Consultoria Ambiental, Rua 01, 371, CEP 89760-000, Itá (SC), Brasil. bia_binotto@hotail.com

2 Engenheira Agrônoma, Florestal Alto Uruguai Ltda., Rua Gentil Miorando, 332, CEP 99700-000, Erechim (RS), Brasil.Ana_antoni@hotmail.com

3 Bióloga, MSc., Programa de Pós-Graduação em Ecologia URI Erechim, Av. Sete de Setembro, 1621, CEP 99700-000, Erechim (RS), Brasil.g_neumann@yahoo.com.br

4 Bióloga, Dra ${ }^{\mathrm{a}}$, Departamento de Ciências Biológicas, Universidade Regional Integrada, Av. Sete de Setembro, 1621, CEP 99700-000, Erechim (RS), Brasil. tasausen@uricer.edu.br

5 Biólogo, Dr., Laboratório de Sistemática e Ecologia Vegetal - ECOSSIS, Departamento de Ciências Biológicas, Universidade Regional Integrada, Av. Sete de Setembro, 1621, CEP 99700-000, Erechim (RS), Brasil.
\end{abstract}

Recebido para publicação em 15/01/2014 e aceito em 5/03/2015

Ci. Fl., v. 26, n. 4, out.-dez., 2016 
growing throughout 90 days of flood recovery. We did not found differences in growth variables and biomass allocation in relation to the control group. However, we observed that plants subjected to 20 days of partial flooding had higher leaf abscission and were accompanied by an increase in the chlorophyll levels. The results indicated that this species shows tolerance to partial flooding and should be carefully selected for planting areas.

Keywords: biomass allocation; ecological restoration; forest seedlings; hydrological regime.

\section{INTRODUÇÃO}

Formações ribeirinhas são áreas transicionais entre os sistemas terrestres e aquáticos, arranjadas em rede na paisagem, incluindo um amplo espectro de elementos biológicos e valores socioeconômicos. Caracterizando-se como sistemas próximos de um equilíbrio dinâmico e promotores de elevada heterogeneidade ambiental, as comunidades biológicas das zonas ripárias frequentemente estão entre as mais produtivas e diversas do globo (NILSSON; SVEDMARK, 2002; BUDKE et al., 2008).

Nem todos os regimes de inundação favorecem a evolução de características particulares que facilitem a sobrevivência a inundações ou secas (LYTLE, 2001). Segundo a teoria de histórias de vida, a magnitude, frequência e previsibilidade dos distúrbios afetam a forma como as populações se adaptam ou são eliminadas por estes eventos. Quando os eventos são frequentes, de grande magnitude e previsíveis, a seleção favorece histórias de vida sincronizadas para evitar ou explorar estes eventos extremos (a chamada zona evolucionária) (JUNK et al., 1989; WALKER et al., 1995). Por outro lado, eventos de distúrbio que são de grande magnitude e frequentes, porém, de baixa previsibilidade geram alta mortalidade sobre as populações (zona ecológica) (LYTLE; POFF, 2004). Ainda, segundo Neiff (1997), a maioria das espécies vegetais tem sincronizado seus ritmos de reprodução (produção e dispersão de sementes) de acordo com a magnitude em que ocorrem as fases hidrológicas, sendo que em muitos casos, a intensidade (altura alcançada pela coluna de água) e a amplitude (segmento de tempo em que o rio permanece na fase de inundação) são os principais atributos do regime hidrológico que diretamente interferem nas respostas vegetais.

Os eventos de inundação geram diferentes impactos sobre as populações de plantas distribuídas em zonas ribeirinhas, incluindo mudanças morfológicas, anatômicas e fisiológicas (KOSLOWSKI, 2002), tais como a formação de lenticelas hipertrofiadas e aerênquima, redução da taxa metabólica e da taxa fotossintética (PAROLIN, 2009), alterações na alocação de biomassa, com um rápido crescimento caulinar como maneira de aumentar a altura e escapar dos efeitos da submersão (PAROLIN, 2001a). O alagamento influencia também as características do solo, principalmente em relação à disponibilidade de oxigênio, reduzindo as trocas gasosas entre solo e o ar e, consequentemente, interferindo em processos do desenvolvimento vegetal. Neste sentido, as respostas das plantas ao alagamento são muito variadas e dependem do estádio de desenvolvimento do indivíduo.

No Brasil, os exemplos clássicos de grandes áreas inundáveis remetem para as várzeas da bacia Amazônica e a planície aluvial do Pantanal, apresentando ritmos sazonais (regulares) de alagamento, com períodos alternados de cheia e seca (JUNK et al., 1989). Por outro lado, nas florestas ribeirinhas do Alto Uruguai, região sul do Brasil, os eventos de inundação podem ser caracterizados como imprevisíveis e de baixa magnitude (reduzida altura da coluna de água e amplitude média menor que 15 dias), comuns no sul do Brasil, podendo ocorrer sucessivas vezes ao longo do ano hidrológico (BUDKE et al., 2010). Nestas formações florestais, Cedrela fissilis Vell. (Meliaceae) apresenta uma ampla distribuição, desenvolvendo-se no interior de florestas primárias, podendo também ser encontrada como espécie pioneira e próxima à margem de rios (OLIVEIRA-FILHO et al., 2006), sobretudo, nas áreas abrangidas pela Floresta Estacional Semidecídua e Floresta Ombrófila Mista (ANTONIAZZI et al., 2013).

A ampla distribuição desta espécie em formações florestais brasileiras, associada ao alto valor agregado à sua madeira, tem compelido muitos trabalhos a indicarem esta espécie para fins de restauração ambiental, sobretudo de florestas ribeirinhas e para reflorestamentos heterogêneos (LORENZI, 2008), bem como para utilização em sistemas agroflorestais e outras aplicações (DURIGAN; NOGUEIRA, 1990). Porém, considerando diferentes regimes de inundação, 
são escassos os trabalhos que indiquem espécies adequadas para fins de restauração em áreas que sofram este tipo de distúrbio. Neste sentido, avaliouse o desenvolvimento, bem como a tolerância de plântulas de Cedrela fissilis a diferentes amplitudes e intensidades de inundação, a partir de suas respostas morfológicas, alocação de biomassa e concentração de pigmentos fotossintetizantes, considerando as seguintes questões: i) a espécie é tolerante a inundação em seus estádios iniciais de desenvolvimento?; ii) caso seja tolerante, esta tolerância ocorre quando toda a planta é inundada ou apenas parcialmente (etapa intensidade da inundação)? e iii) caso seja tolerante, a tolerância ao alagamento ocorre em situações de inundação por rios de pequeno porte, ou seja, nas quais a inundação não ultrapassa uma ou duas semanas (etapa amplitude de inundação)?

\section{MATERIAL E MÉTODOS}

\section{Coleta de sementes e preparação do experimento}

A coleta de frutos de Cedrela fissilis foi realizada em Severiano de Almeida - RS, município com coordenada central $27^{\circ} 20^{\prime} 17^{\prime \prime}$ de latitude sul e $52^{\circ} 01^{\prime} 38^{\prime \prime}$ de longitude oeste. As sementes de Cedrela fissilis germinaram em bandejas contendo solo organo-argiloso e vermiculita na proporção 3:1, alocados no Laboratório de Sistemática e Ecologia Vegetal da Universidade Regional Integrada do Alto Uruguai e das Missões - Campus de Erechim. Quando 75\% das sementes germinaram e as plântulas atingiram cinco centímetros de altura, ocorreu à repicagem e plantio das mesmas em tubetes de polietileno com volume de $100 \mathrm{~cm}^{3}$. Os tubetes foram preenchidos com solo organoargiloso, esterco bovino "maturado" e vermiculita nas proporções 2:1:1, de acordo com metodologia proposta por Fonseca e Ribeiro (1998). Os tubetes permaneceram em casa de vegetação para aclimatação durante 60 dias, recebendo irrigação por 10 minutos, três vezes ao dia. As condições de temperatura do ar, durante o período experimental, foram registradas com data logger (Pinguim, RHT) instalado na casa de vegetação, com temperaturas médias de $28^{\circ} \mathrm{C}$ ao longo do período experimental.

\section{Desenho experimental}

O delineamento experimental foi constituído por dois fatores de análise: (i) intensidade de inundação, com dois níveis (plântulas submersas na altura do colo e plântulas totalmente submersas) e (ii) amplitude de inundação, com três níveis (cinco, 15 e 20 dias de submersão). Cada uma das amplitudes de inundação foi composta por 20 plântulas (réplicas), em cada um dos fatores de intensidade (submersão parcial ou total), totalizando 120 plântulas. Todo o experimento foi simulado em caixa d'água de 500 litros. Um grupo controle consistiu em 20 plântulas que foram mantidas em tubetes de polietileno com volume de $100 \mathrm{~cm}^{3}$, na mesma casa de vegetação, recebendo irrigação três vezes ao dia.

\section{Coleta e análise de dados}

Após o período de inundação, as plântulas foram retiradas dos respectivos tratamentos (intensidade e amplitude de inundação) e foram dispostas em bandejas, juntamente com o grupocontrole, para avaliação do processo de recuperação da inundação. Após 30,60 e 90 dias do término da respectiva inundação, todas as plântulas foram avaliadas quanto à altura da parte aérea, diâmetro do colo, número de folhas/folíolos e índices de clorofila Falker-ICF ( $a, b$ e total) foram obtidos por meio de um medidor eletrônico de clorofila ClorofiLog CFL 1030 (Falker Ltda, Porto Alegre, Brasil), cujas estimativas dos teores de clorofilas estão baseadas nas relações de absorção de diferentes frequências das clorofilas $a, b$ e total.

Uma segunda coleta de dados foi realizada após 90 dias e consistiu na remoção das plântulas dos tubetes e analisadas quanto à altura total (raiz até gema apical), diâmetro da raiz (dois centímetros abaixo do solo) e o comprimento da raiz principal. Também ocorreu a separação morfológica em folhas, caules, e raízes, para obtenção da massa seca. A área foliar foi mensurada em uma folha mediana completamente expandida de cada planta por meio da obtenção de uma imagem digital das folhas com o programa ImageJ 1.6 (FERREIRA; RASBAND, 2012), e expressa em $\mathrm{cm}^{2}$.

As amostras de folhas, caules e raízes foram colocadas em estufa com temperatura de $40 \pm 3^{\circ} \mathrm{C}$ por 48 horas (folhas) e 96 horas (caules e raízes) para determinação da massa seca. Posteriormente, as amostras foram pesadas em balança analítica de precisão. A partir dessas informações foram calculadas a massa seca total e as razões de alocação raiz:parte aérea (R:PA), razão massa de raízes:massa total (RMR) e razão de massa foliar:massa total (RMF). 
O experimento foi conduzido em um delineamento casualizado e os dados coletados foram submetidos à análise de variância (ANOVA) utilizando-se o programa Past (HAMMER; HARPER, 2009). As médias dos tratamentos foram comparadas aos pares pelo teste de Tukey, em um nível de significância de 5\%.

\section{RESULTADOS}

Plântulas de Cedrela fissilis não toleram regimes de inundação total, visto que foi registrada a mortalidade de todas as plântulas neste tratamento (cinco, 15 e 20 dias), já nos cinco dias de inundação. No entanto, a espécie mostrou-se tolerante à inundação parcial (inundação até o colo), com uma média de $81 \%$ de sobrevivência das plântulas, em todas as amplitudes de inundação. Os parâmetros de crescimento (altura da parte aérea e diâmetro do caule) não apresentaram diferenças com o aumento da amplitude de inundação parcial, em todos os períodos de recuperação da inundação $(30,60$ e 90 dias) (Tab. 1, 2 e 3), com exceção do diâmetro do caule, aos 90 dias de recuperação da inundação.

Houve decréscimo no número de folhas e folíolos com o aumento da amplitude de inundação, porém, a espécie conseguiu retomar o crescimento foliar após inundação parcial, observando-se incremento no número de folhas e folíolos após 30, 60 e 90 dias de recuperação da inundação (Tabelas 1, 2 e 3). Quanto aos Índices de Clorofila Falker (ICF), houve um aumento gradativo nos teores de clorofilas $a, b$ e total com o aumento da amplitude de inundação para um mesmo período de recuperação. Ao longo do período de recuperação, observou-se uma redução nos índices de clorofila com o aumento do tempo de recuperação (Tabelas 1, 2 e 3).

A massa seca das folhas, caule e raízes após 90 dias de recuperação da inundação não apresentaram diferença entre as amplitudes de inundação $(p>0,05)$ (Figura 1), bem como para os padrões de alocação de biomassa (RMF, RMR e R:PA) comparado às plântulas controle (Tabela 4). $\mathrm{O}$ comprimento e diâmetro das raízes e a área foliar total das plântulas nas diferentes amplitudes de inundação também não apresentaram diferença em relação às plântulas controle (Figuras 2A, 2B e 2C).

\section{DISCUSSÃO}

Neste trabalho, plântulas de Cedrela fissilis submetidas à inundação parcial não apresentaram redução da altura, área foliar, diâmetro da raiz e comprimento da raiz. Alguns autores (PEZESHKI, 1993; PEZESHKI et al., 1993; DREYER, 1994; LIAO; LIN, 1996) observaram tanto em espécies

TABELA 1: Variáveis morfométricas em plantas de Cedrela fissilis submetidas à inundação parcial em diferentes amplitudes (cinco, 15 e 20 dias) e após a recuperação em casa de vegetação por 30 dias. Os valores são médias seguidas pelo erro padrão da média a partir de ANOVA. ICF = Índice de Clorofila Falker.

TABLE 1: Morphometric variables in Cedrela fissilis plants subjected to partial flooding at different amplitudes (five, 15 and 20 days) and after recovery in the greenhouse for 30 days. Values are means followed by the standard errors from ANOVA. ICF = Falker Chlorophyll Index.

\begin{tabular}{lccccc}
\hline \multicolumn{1}{c}{ Parâmetros } & Controle & 5 dias & 15 dias & 20 dias & $\mathrm{F}$ \\
\hline Altura aérea $(\mathrm{cm})$ & $12,1 \pm 2,0$ & $11,8 \pm 1,4$ & $12,6 \pm 2,0$ & $12,2 \pm 1,9$ & $0,59 \mathrm{~ns}$ \\
Diâmetro $(\mathrm{mm})$ & $4,2 \pm 0,5$ & $4,9 \pm 0,9$ & $4,2 \pm 1,3$ & $4,2 \pm 1,0$ & $2,57 \mathrm{~ns}$ \\
$\mathrm{~N}^{\circ}$ folhas & $8,8 \pm 2,1 \mathrm{ab}$ & $9,5 \pm 2,2 \mathrm{a}$ & $6,8 \pm 3,7 \mathrm{~b}$ & $6,5 \pm 2,2 \mathrm{~b}$ & $5,99^{*}$ \\
$\mathrm{~N}^{\circ}$ folíolos & $54,0 \pm 7,0 \mathrm{ab}$ & $62,8 \pm 13,5 \mathrm{a}$ & $48,5 \pm 22,9 \mathrm{~b}$ & $42,5 \pm 12,2 \mathrm{~b}$ & $6,40^{*}$ \\
$\mathrm{ICF} a$ & $23,2 \pm 3,9 \mathrm{ab}$ & $22,2 \pm 3,5 \mathrm{a}$ & $23,0 \pm 5,7 \mathrm{ab}$ & $25,2 \pm 5,9 \mathrm{~b}$ & $2,59^{*}$ \\
ICF $b$ & $5,0 \pm 1,4 \mathrm{ab}$ & $4,6 \pm 1,1 \mathrm{~b}$ & $5,0 \pm 1,8 \mathrm{ab}$ & $5,7 \pm 2,1 \mathrm{a}$ & $2,73^{*}$ \\
ICF total & $28,2 \pm 5,2 \mathrm{ab}$ & $26,8 \pm 4,5 \mathrm{~b}$ & $27,9 \pm 7,5 \mathrm{ab}$ & $30,9 \pm 8,0 \mathrm{a}$ & $2,67^{*}$ \\
\hline
\end{tabular}

Em que: ns $=$ não significativo; $*=p<0,05$ 
sensíveis como em espécies tolerantes a este tipo de distúrbio, a redução da área foliar, abscisão, senescência e redução na produção de novas folhas. Entretanto, no presente estudo foi verificado que Cedrela fissilis parece resistir à inundação parcial, embora algumas plântulas tenham apresentado sintomas característicos de espécies sensíveis, como clorose e senescência foliar. O desenvolvimento do sistema radicular avaliado através do diâmetro e o comprimento da raiz principal não foram

TABELA 2: Variáveis morfométricas em plantas de Cedrela fissilis submetidas à inundação parcial em diferentes amplitudes (cinco, 15 e 20 dias) e após a recuperação em casa de vegetação por 60 dias. Os valores são médias seguidas pelo erro padrão da média a partir de ANOVA. ICF $=$ Índice de Clorofila Falker.

TABLE 2: Morphometric variables in Cedrela fissilis plants subjected to partial flooding at different amplitudes (five, 15 and 20 days) and after recovery in the greenhouse for 60 days. Values are means followed by the standard errors from ANOVA. ICF = Falker Chlorophyll Index.

\begin{tabular}{lccccc}
\hline \multicolumn{1}{c}{ Parâmetros } & Controle & 5 dias & 15 dias & 20 dias & $\mathrm{F}$ \\
\hline Altura aérea (cm) & $13,7 \pm 2,0$ & $13,0 \pm 1,4$ & $13,6 \pm 2,5$ & $13,8 \pm 1,9$ & $0,64 \mathrm{~ns}$ \\
Diâmetro (mm) & $5,0 \pm 0,6$ & $5,8 \pm 1,0$ & $5,6 \pm 1,5$ & $5,3 \pm 1,0$ & $2,61 \mathrm{~ns}$ \\
$\mathrm{~N}^{\circ}$ folhas & $8,1 \pm 2,8 \mathrm{ab}$ & $9,8 \pm 2,9 \mathrm{a}$ & $8,1 \pm 2,9 \mathrm{ab}$ & $6,9 \pm 2,0 \mathrm{~b}$ & $3,49^{*}$ \\
$\mathrm{~N}^{\circ}$ folíolos & $66,6 \pm 13,5 \mathrm{ab}$ & $83,1 \pm 22,1 \mathrm{a}$ & $70,8 \pm 22,9 \mathrm{ab}$ & $57,7 \pm 17,7 \mathrm{~b}$ & $5,48^{*}$ \\
ICF $a$ & $24,0 \pm 5,3 \mathrm{bc}$ & $21,5 \pm 3,7 \mathrm{c}$ & $25,0 \pm 5,2 \mathrm{~b}$ & $28,0 \pm 4,6 \mathrm{a}$ & $11,25^{*}$ \\
ICF $b$ & $4,8 \pm 1,6 \mathrm{bc}$ & $4,4 \pm 1,2 \mathrm{c}$ & $5,4 \pm 1,9 \mathrm{~b}$ & $6,5 \pm 1,7 \mathrm{a}$ & $12,05^{*}$ \\
ICF total & $28,8 \pm 6,6 \mathrm{bc}$ & $25,8 \pm 4,7 \mathrm{c}$ & $30,4 \pm 7,0 \mathrm{~b}$ & $34,5 \pm 6,3 \mathrm{a}$ & $11,89^{*}$ \\
\hline
\end{tabular}

Em que: $\mathrm{ns}=$ não significativo; ${ }^{*}$ significativo a $5 \%(p<0,05)$.

TABELA 3: Variáveis morfométricas em plantas de Cedrela fissilis submetidas à inundação parcial em diferentes amplitudes (cinco, 15 e 20 dias) e após a recuperação em casa de vegetação por 90 dias. Os valores são médias seguidas pelo erro padrão da média a partir de ANOVA. ICF $=$ Índice de Clorofila Falker.

TABLE 3: Morphometric variables in Cedrela fissilis plants subjected to partial flooding at different amplitudes (five, 15 and 20 days) and after recovery in the greenhouse for 90 days. Values are means followed by the standard errors from ANOVA. ICF $=$ Chlorophyll Index Falker.

\begin{tabular}{lccccc}
\hline \multicolumn{1}{c}{ Parâmetros } & Controle & 5 dias & 15 dias & 20 dias & $\mathrm{F}$ \\
\hline Altura aérea $(\mathrm{cm})$ & $14,9 \pm 2,3$ & $14,3 \pm 1,5$ & $15,7 \pm 2,1$ & $15,0 \pm 1,6$ & $1,30 \mathrm{~ns}$ \\
Diâmetro $(\mathrm{mm})$ & $5,8 \pm 0,7 \mathrm{~b}$ & $6,9 \pm 1,0 \mathrm{a}$ & $7,1 \pm 1,1 \mathrm{a}$ & $6,9 \pm 1,0 \mathrm{a}$ & $6,97^{*}$ \\
$\mathrm{~N}^{\circ}$ folhas & $7,3 \pm 2,8$ & $8,8 \pm 1,9$ & $8,0 \pm 2,1$ & $7,4 \pm 2,5$ & $1,68 \mathrm{~ns}$ \\
$\mathrm{~N}^{\circ}$ folíolos & $63,7 \pm 16,6 \mathrm{~b}$ & $84,7 \pm 17,9 \mathrm{a}$ & $75,9 \pm 19,6 \mathrm{ab}$ & $67,0 \pm 23,3 \mathrm{~b}$ & $4,53^{*}$ \\
ICF $a$ & $18,2 \pm 6,7 \mathrm{~b}$ & $18,7 \pm 4,0 \mathrm{~b}$ & $21,0 \pm 4,5 \mathrm{~b}$ & $24,3 \pm 4,2 \mathrm{a}$ & $10,46^{*}$ \\
ICF $b$ & $4,7 \pm 1,4 \mathrm{a}$ & $3,7 \pm 0,9 \mathrm{~b}$ & $4,4 \pm 1,3 \mathrm{ab}$ & $4,6 \pm 1,5 \mathrm{a}$ & $3,90^{*}$ \\
ICF total & $22,9 \pm 7,6 \mathrm{~b}$ & $22,4 \pm 4,7 \mathrm{~b}$ & $25,4 \pm 5,8 \mathrm{ab}$ & $28,9 \pm 5,5 \mathrm{a}$ & $8,27^{*}$ \\
\hline
\end{tabular}

Em que: $\mathrm{ns}=$ não significativo; $*=p<0,05$ 


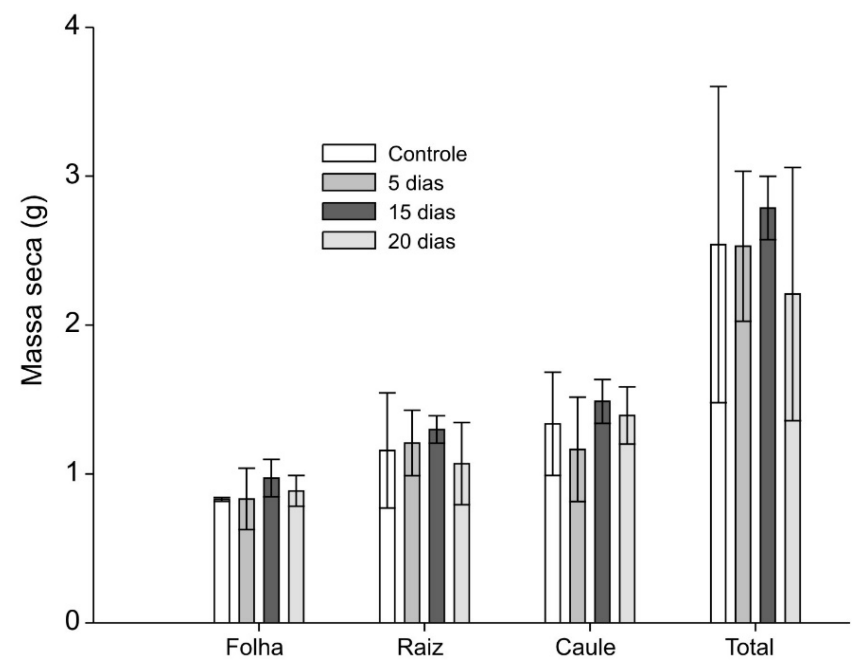

FIGURA 1: Massa seca de folhas, caules, raízes e total em plântulas de Cedrela fissilis submetidas a diferentes amplitudes (cinco, 15 e 20 dias) de inundação e mantidas em recuperação por 90 dias em casa de vegetação. Os valores representam as médias e a barra representa o erro padrão da média a partir de ANOVA.

FIGURE 1: Leaf, stem and root dry mass of Cedrela fissilis seedlings subjected to partial flooding at different amplitudes (five, 15 and 20 days) and after recovery in the greenhouse for 90 days. Values represent averages and bars refers to standard errors from ANOVA.

TABELA 4: Parâmetros de alocação de biomassa entre folhas (F:BT) e raízes (R:BT) e a biomassa total e relação raiz: parte aérea (R:PA) em plantas de Cedrela fissilis submetidas à inundação parcial em diferentes amplitudes e após 90 dias de recuperação da inundação.

TABLE 4: Parameters of biomass allocation between leaves (F: BT) and roots (R BT) and total biomass and root: shoot (R: S) in Cedrela fissilis plants subjected to partial flooding at different amplitudes and after 90 days of flood recovery.

\begin{tabular}{cccccc}
\hline Parâmetros & Controle & 5 dias & 15 dias & 20 dias & F \\
\hline F:BT & $0,2 \pm 0,07$ & $0,2 \pm 0,02$ & $0,2 \pm 0,01$ & $0,2 \pm 0,02$ & $0,59 \mathrm{~ns}$ \\
R:BT & $0,3 \pm 0,04$ & $0,4 \pm 0,07$ & $0,3 \pm 0,02$ & $0,4 \pm 0,02$ & $0,82 \mathrm{~ns}$ \\
R:PA & $0,6 \pm 0,1$ & $0,8 \pm 0,2$ & $0,6 \pm 0,05$ & $0,7 \pm 0,06$ & $0,88 \mathrm{~ns}$ \\
\hline
\end{tabular}

prejudicados com a inundação parcial, visto que não foram observadas diferenças entre plântulas inundadas e plântulas controle.

Marques et al. (1996), em um estudo com plântulas de Cedrela fissilis em inundação parcial durante 60 dias, demonstraram que a hipoxia do solo causa inibição no crescimento das plantas, característica de espécies com baixa ou nenhuma tolerância a esta condição limitante. Contudo, neste estudo não foi observado uma redução no crescimento das plântulas, provavelmente devido ao menor tempo em que estas foram submetidas à inundação parcial (cinco, 15 e 20 dias). A ausência de diferença entre os parâmetros avaliados pode estar associada ao pequeno intervalo de tempo entre as diferentes amplitudes de inundação, em comparação a outros trabalhos realizados em períodos superiores a 30 dias de alagamento. Dentre estes, destaca-se o estudo realizado por Medri et al. (2007), com plântulas de Lithraea molleoides submetidas a um período de 35 dias de alagamento do substrato, assim como Medri et al. (2011), ao avaliarem o efeito do alagamento sobre indivíduos jovens de Aegiphila sellowiana, mantidos em substrato drenado e alagado por 50 e 80 dias.

O contínuo crescimento da altura da parte aérea e do diâmetro do caule observado nas plântulas submetidas às diferentes amplitudes de inundação 

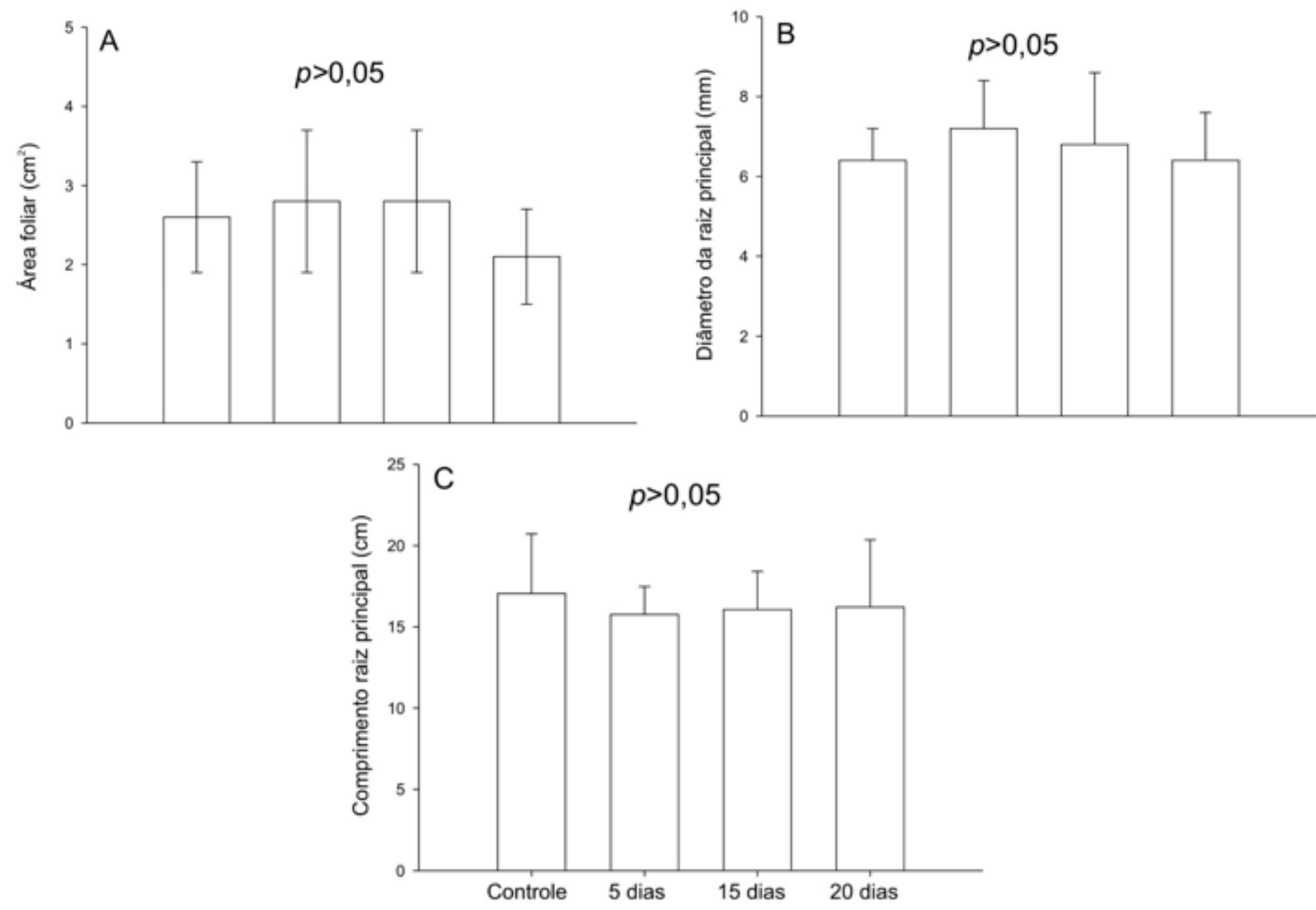

FIGURA 2: Área foliar (A), diâmetro da raiz principal (B) e comprimento da raiz principal (C) em plântulas de Cedrela fissilis submetidas à inundação parcial em diferentes amplitudes (cinco, 15 e 20 dias). Os valores representam as médias e a barra representa o erro padrão da média a partir de ANOVA.

FIGURE 2: Leaf area (A), diameter of the main root (B) and length of the main root (C) in Cedrela fissilis seedlings subjected to partial flooding at different amplitudes (five, 15 and 20 days). Values represent averages and bars refers to standard errors from ANOVA.

e durante os 90 dias de recuperação sugere que o desenvolvimento das plântulas não foi afetado pela inundação parcial. Resultado semelhante foi observado em plantas de Mauritia vinifera Mart. (CALBO et al., 1998) e Copernicia prunifera (Mill.) H. E. Moore, (ARRUDA; CALBO, 2004). Entretanto, em Sebastiania commersoniana (Baill.) L. B. Sm. \& Downs (KOLB et al., 1998) e Genipa americana L. (MIELKE et al., 2003) foi observado um decréscimo acentuado no crescimento, chegando a níveis irreversíveis, ou seja, não permitindo a retomada do crescimento após a drenagem do solo.

A manutenção do crescimento em condições de hipoxia do solo depende do estado nutricional das plantas e do crescimento em altura. Estas características podem ser fundamentais para a sobrevivência da planta, visto que o aumento na altura da parte aérea pode permitir o escape dos efeitos da submersão total (SANTIAGO; PAOLI,
2003; PAROLIN, 2001b), bem como de outros fatores normalmente associados às respostas do crescimento, como o nível da água acima do solo e o tempo de duração do alagamento (PAROLIN. 2001b). Nos diferentes tempos da inundação parcial, observou-se que algumas plântulas apresentaram clorose e senescência foliar, apesar da baixa taxa de abscisão foliar, o que pode estar associado com a redução na disponibilidade de nutrientes minerais no substrato (ISHIDA et al., 2002).

É importante ressaltar que durante os períodos de recuperação as plântulas apresentaram uma tendência geral de diminuição da abscisão foliar acompanhada por um aumento na produção de folhas e folíolos. Todavia, a produção de novas folhas e o acúmulo de clorofila apresentou um padrão oposto. As plântulas submetidas a regimes de inundação parcial por 20 dias apresentaram um menor número de folhas e maior índice de clorofila 
em relação às plântulas sob inundação parcial por cinco e 15 dias e plântulas controle. Este padrão pode estar associado com uma maior degradação de pigmentos e reabsorção de nutrientes nas plântulas submetidas ao maior tempo de inundação parcial (15 dias) antes da abscisão foliar. Por outro lado, nas plântulas submetidas a menores períodos de inundação (cinco dias), o menor índice de clorofila pode estar associado com uma menor concentração de clorofila nas folhas novas.

$\mathrm{O}$ alagamento provocou o aparecimento de lenticelas hipertrofiadas em algumas plântulas, a partir de cinco dias de inundação parcial, indicando uma importante resposta de tolerância à hipoxia, normalmente associada a solos alagados. Em plântulas de Peltophorum dubium (Benth.) Brenan, foi observado que o desenvolvimento de lenticelas permitiu a entrada de oxigênio atmosférico, que se difundiu da parte aérea para as raízes (PIMENTA et al., 1998). Outros trabalhos de Ferreira et al. (2001) comprovaram que a produção de lenticelas hipertrofiadas em plântulas inundadas de Piptadenia gonoacantha (Mart.) J. F. Macbr., poderia ser interpretada como uma resposta adaptativa, a fim de aumentar o transporte de $\mathrm{O}_{2}$ dos tecidos aéreos para as raízes, conforme demonstrado experimentalmente por Lobo e Joly (1995) em plântulas de Magnolia ovata (A. St.-Hil.) Spreng.

Lobo e Joly (1998) sugerem que a capacidade de manter ou aumentar a massa seca da parte aérea durante períodos de inundação similares aos seus habitat naturais pode ser utilizado como um critério para classificar as espécies tolerantes à inundação. Conforme esse critério, o experimento realizado com plântulas de Cedrela fissilis demonstrou que a espécie pode ser classificada como tolerante à inundação parcial, visto que não foram observadas diferenças no acúmulo de massa seca nas folhas, caule e raízes entre as plântulascontrole e em diferentes amplitudes de inundação, bem como nos padrões de alocação de biomassa.

Por outro lado, Medri et al. (2002) evidenciaram, em várias espécies, um efeito negativo do alagamento sobre o crescimento e o desenvolvimento da planta como um todo, mesmo em muitas espécies consideradas tolerantes. Essas observações ressaltam a necessidade de critérios múltiplos para categorizar as plantas como tolerantes ou sensíveis, uma vez que o próprio decréscimo em algum parâmetro pode estar associado com o desenvolvimento de alguma estratégia adaptativa para tolerar a inundação, como a relação observada entre o número de folhas e o índice de clorofila nas plântulas sob inundação.

\section{CONCLUSÕES}

Para Cedrela fissilis, a inundação total mostra-se como situação crítica, com o registro de morte de todas as plântulas a partir da amplitude de cinco dias de inundação.

Os resultados observados neste estudo confirmam a indicação da espécie como "acompanhante ciliar", ou seja, com ocorrência em florestas ribeirinhas, em solos úmidos, porém, sem inundação constante, uma situação frequente no sul do Brasil, em especial, em ambientes submetidos ao encharcamento do solo, mas não completamente inundados. Desta forma, o cedro representa uma interessante opção para plantio em ambientes que não sofram inundações de grande intensidade, sobretudo, em ambientes com encharcamento eventual.

\section{AGRADECIMENTOS}

Ao Laboratório de Sistemática e Ecologia Vegetal - ECOSSIS da Universidade Regional Integrada do Alto Uruguai e das Missões - URI, Campus de Erechim, pelo apoio financeiro e logístico. B. Binotto e A.P. Antoniazzi receberam bolsas do programa REDES-URI. G.M. Neumann recebeu bolsa PROSUP-CAPES. Aos revisores anônimos, pelas sugestões e recomendações.

\section{REFERÊNCIAS BIBLIOGRÁFICAS}

ANTONIAZZI, A. P. et al. Eficiência de recipientes no desenvolvimento de mudas de Cedrela fissilis Vell. (Meliaceae). Revista Brasileira de Biociências, Porto Alegre, v. 11, p. 313-317, 2013. ARRUDA, G. M. T.; CALBO, M. E. R. Efeitos da inundação no crescimento, trocas gasosas e porosidade radicular da carnaúba (Copernicia prunifera (Mill.) H. E. Moore). Acta Botanica Brasilica, Porto Alegre, v. 18, p. 219-224, 2004.

BUDKE, J. C. et al. Tree community features of two stands of riverine forest under different flooding regimes in Southern Brazil. Flora, London, v. 203, p. 162-174, 2008.

BUDKE, J. C. et al. Florestas ribeirinhas e inundações: de contínuos espaciais a gradientes temporais. In: SANTOS, J. E.; ZANIN, E. M.; MOSCHINI, L.E. (Orgs.). Faces da polissemia da 
paisagem: ecologia, planejamento e percepção. São Carlos: Rima, 2010. v. 3. p. 201-218.

CALBO, M. E. R. et al. Crescimento, condutância estomática, fotossíntese e porosidade do buriti sob inundação. Revista Brasileira de Fisiologia Vegetal, Londrina, v. 10, p. 51-58, 1998.

DREYER, E. Compared sensitivity of seedlings from 3 woody species (Quercus rubra L. and Fagus silvatica L.) to waterlogging and associated root hypoxia: effects on water relations and photosynthesis. Annals of Forest Science, Paris, v. 51, p. 417-429, 1994.

DURIGAN, G.; NOGUEIRA, J. C. B. Recomposição de matas ciliares. São Paulo: Instituto Florestal, 1990.

FERREIRA, N. J. et al. Crescimento inicial de Piptadenia gonoacantha (Leguminosae, Mimosoideae) sob inundação em diferentes níveis de luminosidade. Revista Brasileira de Botânica, São Paulo, v. 24, p. 561-566, 2001.

FERREIRA, T.; RASBAND, W. S. ImageJ 1.46r user guide. U. S. National Institutes of Health, Maryland: Bethesda, 2012.

FONSECA, C. E. L.; RIBEIRO, J. F. Produção de mudas e crescimento inicial de espécies arbóreas. In: RIBEIRO, J. F. (Org.). Cerrado: matas ciliares de galeria. Planaltina: Embrapa, 1998. p. 119-133. HAMMER, O.; HARPER, D. A. T. PAST version 2.06. 2009. Disponível em: $<\mathrm{http} / / /$ folk.uio.no/ ohammer/past>. Acesso em: 20 maio 2011.

JUNK, W. J. et al. The flood pulse concept in riverfloodplain systems. Canadian Publication of Fisheries and Aquatic Sciences, Ottawa, v. 106, p. 110-127, 1989.

KOLB, R. M. et al. Anatomia ecológica de Sebastiania commersoniana (Baill.) L.B. Sm. \& Downs (Euphorbiaceae) submetida ao alagamento. Revista Brasileira de Botânica, São Paulo, v. 21, n. 3, p. 305-312, 1998.

KOSLOWSKI, T. T. Physiological-ecological impacts of flooding on riparian forest ecosystems. Wetlands, New York, v. 22, p. 550-561, 2002.

LIAO, C. T ; LIN, C. H. Photosynthetic responses of grafted bitter melon seedlings to flood stress. Environmental and Experimental Botany, New York, v. 36, p. 167-172, 1996.

LOBO, P. C.; JOLY, C. A. Tolerance to hypoxia and anoxia in neotropical tree species. In: SCARANO, F. R.; FRANCO, A. C. (Eds.). Ecophysiological strategies of xerophytic and amphibious plants in the neotropics. Rio de Janeiro: UFRJ, 1998. p. 137-156. (Series Oecologia Brasiliensis, v. 4).
LOBO, P. C.; JOLY, C. A. Mecanismos de tolerância à inundação de plantas de Talauma ovata St. Hil. (Magnoliaceae), uma espécie típica de matas de brejo. Revista Brasileira de Botânica, São Paulo, v. 18, p. 177-184, 1995.

LORENZI, H. Árvores brasileiras: manual de identificação e cultivo de plantas arbóreas nativas do Brasil. Nova Odessa: Instituto Plantarum, 2008. LYTLE, D. A. Disturbance regimes and life history evolution. American Naturalist, Chicago, v. 157, p. 525-536, 2001.

LYTLE, D. A.; POFF, N. L. Adaptation to natural flow regimes. Trends in Ecology and Evolution, Amsterdam, v. 19, p. 94-100, 2004.

MARQUES, M. C. M. et al. Aspectos do metabolismo e da morfologia de Cedrela fissilis Vell. e Anadenanthera colubrina (Vell.) Bren. submetidas a diferentes regimes hídricos. Brazilian Archives of Biology and Technology, Curitiba, v. 39, p. 385-392, 1996.

MEDRI, C. et al. Morfoanatomia de órgãos vegetativos de plantas juvenis de Aegiphila sellowiana Cham. (Lamiaceae) submetidas ao alagamento do substrato. Acta Botanica Brasilica, Porto Alegre, v. 25, p. 445-454, 2011.

MEDRI, M. E. et al. Estudos sobre a tolerância ao alagamento em espécies arbóreas nativas da bacia do rio Tibagi. In: SCARANO, F. R.; FRANCO, A. C. et al (Eds.). A Bacia do Rio Tibagi. Londrina: Edição dos Editores, 2002. p. 133-172.

MEDRI, M. E. et al. Alterações morfoanatômicas em plantas de Lithraea molleoides (Vell.) Engl. submetidas ao alagamento. Acta Scientiarum: Biological Sciences, Maringá, v. 29, p. 15-22, 2007. MIELKE, M. S. et al. Leaf gas exchange, chlorophyll fluorecence and growth responses of Genipa americana seedlings to soil flooding. Environmental and Experimental Botany, New York, v. 50, p. 221-231, 2003.

NEIFF, J. J. El régimen de pulsos en ríos y grandes humedales de Sudamérica. In: MALVAREZ, A.I.; KANDUS, P. (Eds.). Tópicos sobre grandes humedales sudamericanos. Montevideo: ORCYTMAB/UNESCO, 1997. p. 99-149

NILSSON, C.; SVEDMARK, M. Basic principles and ecological consequences of changing water regimes: riparian plant communities. Environmental Management, New York, v. 30, p. 468-480, 2002.

OLIVEIRA-FILHO, A. T. et al. Floristic relationships of seasonally dry forests of eastern South America based on tree species distribution 
patterns. In: PENNINGTON, R. T.; LEWIS, G. P.; RATTER, J. A. (Eds.). Neotropical Savannas and Dry forests: plant diversity, biogeography and conservation. Boca Raton: CRC Press, 2006. p. 159-192.

PAROLIN, P. Senna reticulata, a pioneer tree from Amazonian várzea floodplains. The Botanical Review, New York, v. 67, p. 239-254, 2001 a.

PAROLIN, P. Morphological and physiological adjustments to waterlogging and drought in seedlings of Amazonian floodplain trees. Oecologia, Berlin, v. 128, p. 326-335, 2001b.

PAROLIN, P. Submerged in darkness: adaptations to prolonged submergence by woody species of the Amazonian floodplain. Annals of Botany, London, v. 103, p. 359-376, 2009.

PEZESHKI, S. R. Differences in patterns of photosynthetic responses to hypoxia in flood-tolerant and flood-sensitive tree species. Photosynthetica, Prague, v. 28, p. 423-430, 1993.
PEZESHKI, S. R. et al. The influence of soil oxygen deficiency on alcohol dehydrogenase activity, root porosity, ethylene production and photosynthesis in Spartina patens. Environmental and Experimental Botany, New York, v. 33, p. 65-573, 1993.

PIMENTA, J. A. et al. Adaptations to flooding by tropical trees: morphological and anatomical modifications. Oecologia Brasiliensis, Rio de Janeiro, v. 4, p. 157-176, 1998.

SANTIAGO, E. F.; PAOLI, A. A. S. O aumento em superfície em Adelia membranifolia (Müll. Arg.) Pax \& K. Hoffm. e Peltophorum dubium (Spreng.) Taub., em resposta ao estresse por deficiência nutricional e alagamento do substrato. Revista Brasileira de Botânica, São Paulo, v. 26, n. 4, p. 503-513, 2003.

WALKER, K. F. et al. A perspective on dryland river ecosystems. Regulated Rivers: Research and Management, Inglaterra, v. 11, p. 85-104, 1995. 